PROCEEDINGS OF THE

AMERICAN MATHEMATICAL SOCIETY

Volume 128, Number 2, Pages 527-531

$\mathrm{S}$ 0002-9939(99)05136-9

Article electronically published on July 8, 1999

\title{
ON THE INTERMEDIATE INTEGRAL FOR MONGE-AMPÈRE EQUATIONS
}

\author{
JEANNE NIELSEN CLELLAND
}

(Communicated by Lesley M. Sibner)

\begin{abstract}
Goursat showed that in the presence of an intermediate integral, the problem of solving a second-order Monge-Ampère equation can be reduced to solving a first-order equation, in the sense that the generic solution of the first-order equation will also be a solution of the original equation. An attempt by Hermann to give a rigorous proof of this fact contains an error; we show that there exists an essentially unique counterexample to Hermann's assertion and state and prove a correct theorem.
\end{abstract}

The purpose of this paper is to correct an error in the literature regarding Goursat's method of the intermediate integral for Monge-Ampère equations. Recall that a Monge-Ampère equation is a second-order PDE of the form

$$
A\left(z_{x x} z_{y y}-z_{x y}^{2}\right)+B z_{x x}+2 C z_{x y}+D z_{y y}+E=0
$$

where the coefficients $A, B, C, D, E$ are functions of the five variables $x, y, z, z_{x}, z_{y}$. Such an equation can be described geometrically as follows: let $M=\mathbb{R}^{5}$ with coordinates $x, y, z, p, q$, and consider the differential ideal $\mathcal{I}$ on $M$ generated by the forms

$$
\begin{gathered}
\theta=d z-p d x-q d y \\
d \theta=-d p \wedge d x-d q \wedge d y \\
\Omega=A d p \wedge d q+B d p \wedge d y+C(d x \wedge d p+d q \wedge d y)+D d x \wedge d q+E d x \wedge d y
\end{gathered}
$$

The coefficients $A, B, C, D, E$ are now thought of as functions of the variables $x, y, z, p, q$. 2-dimensional submanifolds $i: N^{2} \hookrightarrow M$ satisfying $i^{*}(\theta)=i^{*}(d \theta)=$ $i^{*}(\Omega)=0, i^{*}(d x \wedge d y) \neq 0$ are in one-to-one correspondence with solutions of (1).

This motivates the following geometric definition.

Definition 1. A Monge-Ampère system on a 5-manifold $M^{5}$ is a differential ideal $\mathcal{I} \subset \Omega^{*}(M)$ which is generated by a contact 1 -form $\theta$ (i.e., a 1 -form $\theta$ with the property that $\theta \wedge(d \theta)^{2}$ never vanishes on $M$ ), its exterior derivative $d \theta$, and another 2 -form $\Omega$ with the property that $\Omega$ and $d \theta$ are linearly independent modulo $\theta$ at each point of $M$.

Received by the editors April 6, 1998.

1991 Mathematics Subject Classification. Primary 35A30; Secondary 58A15.

Key words and phrases. Method of the intermediate integral, Monge-Ampère equations, exterior differential systems.

This research was supported in part by NSF grant DMS-9627403.

(C)1999 American Mathematical Society 
This definition includes systems that arise from Monge-Ampère equations as described above, as well as more general systems. An integral manifold of a Monge-Ampère system is a 2-dimensional submanifold $i: N^{2} \hookrightarrow M$ such that $i^{*}(\theta)=i^{*}(d \theta)=i^{*}(\Omega)=0$. If the system arises from a PDE as above, then solutions of the PDE are typically in one-to-one correspondence with those integral manifolds of $\mathcal{I}$ that satisfy a certain independence condition. However, in some contexts more general integral manifolds may be of interest as well. For any MongeAmpère system, there always exist local coordinates $x, y, z, p, q$ on $M$ in which $\mathcal{I}$ takes the form described above, although there may not be a global coordinate expression of this form. In terms of the PDE (1), linear independence of $\Omega$ and $d \theta$ modulo $\theta$ means that the coefficients $A, B, C, D, E$ never vanish simultaneously on $M$. Further discussion of Monge-Ampère systems may be found in [2].

The 2 -form $\Omega$ may be replaced by any expression of the form $\Omega+\lambda d \theta$ without changing the ideal $\mathcal{I}$. Suppose that $\lambda$ is chosen so that the resulting form $\Omega$ is decomposable; i.e., that there exist two 1 -forms $\omega^{1}, \omega^{2}$ such that

$$
\Omega=\omega^{1} \wedge \omega^{2} .
$$

There is a unique (up to nonzero multiples) such choice for $\Omega$ in the case that $\mathcal{I}$ represents a parabolic equation, and there are exactly two distinct such choices for $\Omega$ in the case that $\mathcal{I}$ represents a hyperbolic equation (or an elliptic equation if the forms $\omega^{1}, \omega^{2}$ are allowed to assume complex values).

Having chosen $\Omega$ as above, let $\mathcal{J}$ be the differential system defined by

$$
\mathcal{J}=\left\{\theta, \omega^{1}, \omega^{2}\right\} .
$$

$\mathcal{J}$ is called a characteristic system of $\mathcal{I}$, and the integral curves of $\mathcal{J}$ are characteristics of the system $\mathcal{I}$. This definition agrees with the usual notion of characteristics.

Now suppose that there exists a function $F$ on $M$ such that $d F \in \mathcal{J}$. Goursat [3] calls such a function a first-order intermediate integral for $\mathcal{I}$. The reason for this terminology is as follows: let $F$ be given in local coordinates as $F(x, y, z, p, q)$, and suppose that $N^{2} \subset M$ is an integral manifold of the system $\mathcal{K}$ defined by $\{\theta, d \theta, F\}$. If $N$ satisfies the independence condition $d x \wedge d y \neq 0$, then it represents a solution of the first-order PDE

$$
F\left(x, y, z, z_{x}, z_{y}\right)=0 .
$$

Goursat shows that generically, any such $N$ is in fact an integral manifold of $\mathcal{I}$. Goursat does allow for the possibility of exceptional $N$ that do not have this property ("Le raisonnement ne pourrait être en défaut que si, pour l'intégrale considérée, un des facteurs $\lambda, \nu, \mu$ devenait indéterminé," [3], p. 60).

From the point of view of PDEs, this result gives a method for finding solutions to equation (1): first find an intermediate integral $F$, and then solve the first-order PDE (2). Any solution of (2) should be a solution of the original equation (1). Goursat uses this method to show, for example, that the solutions of the equation

$$
z_{x x} z_{y y}-z_{x y}^{2}=0
$$

are exactly the developable surfaces.

In [4], Hermann attempts to give a rigorous proof of Goursat's result for an arbitrary integral manifold of $\mathcal{K}$, but the proof contains an error. In the remainder of the paper we will discuss Hermann's argument and the error it contains, describe counterexamples, and state and prove a correct theorem.

Theorem 8.1 of [4] may be stated in local coordinates as follows: 
Assertion 2. Suppose that $F$ is an intermediate integral for $\mathcal{I}$ such that $d F$ never vanishes on $M$. Let $i: N^{2} \hookrightarrow M$ be an integral manifold of $\mathcal{K}$ that satisfies the independence condition $i^{*}(d x \wedge d y) \neq 0$. Then $N$ is an integral manifold of $\mathcal{I}$.

The independence condition actually does not enter into the proof, and it is convenient to omit this hypothesis in what follows so that we may consider equivalence of systems under contact transformations. Two Monge-Ampère systems $(M, \mathcal{I}),(\bar{M}, \overline{\mathcal{I}})$ are said to be contact equivalent if there is a diffeomorphism $\psi$ : $M \rightarrow \bar{M}$ such that $\psi^{*} \overline{\mathcal{I}}=\mathcal{I}$. This is a natural notion of equivalence for differential systems, but such transformations do not necessarily preserve independence conditions.

The argument given in [4] is as follows: since $F$ is an intermediate integral of $\mathcal{I}$, we have

$$
d F=a \theta+b \omega^{1}+c \omega^{2}
$$

where by hypothesis $a, b, c$ never vanish simultaneously. The hypotheses also imply that $i^{*}(\theta)=i^{*}(d F)=0$; therefore,

$$
i^{*}\left(b \omega^{1}+c \omega^{2}\right)=0 .
$$

The only way that $N$ can fail to be an integral manifold of $\mathcal{I}$ is if $i^{*}\left(\omega^{1} \wedge \omega^{2}\right) \neq 0$, and by (4) this can only happen if $b=c=0$. But taking the exterior derivative of both sides of (3) shows that this is impossible unless we also have $a=0$, which is a contradiction.

The error is in this last step. The conclusion would be true if we knew that $b=c=0$ held on all of $M^{5}$, but in fact we only know that $i^{*}(b)=i^{*}(c)=0$, i.e., that $b$ and $c$ vanish on a 2-dimensional submanifold of $M$. This is perfectly possible; a counterexample (admittedly a very degenerate one) to the assertion is as follows: take $M=\mathbb{R}^{5}, \omega^{1}=d x, \omega^{2}=d y, F=z$, and let $i: N^{2} \hookrightarrow M$ be the surface defined by $z=p=q=0$. Then

$$
d F=\theta+p \omega^{1}+q \omega^{2}
$$

and $N$ is an integral manifold of $\mathcal{K}$, but $i^{*}\left(\omega^{1} \wedge \omega^{2}\right)=d x \wedge d y \neq 0$, so $N$ is not an integral manifold of $\mathcal{I}$.

Remark. As described above, $\mathcal{I}$ represents the equation $1=0$, which is not exactly an interesting partial differential equation. But in the coordinate system $(X, Y, Z, P, Q)$ defined by

$$
X=p, \quad Y=q, \quad Z=z-p d x-q d y, \quad P=-x, \quad Q=-y,
$$

$\mathcal{I}$ represents the equation

$$
Z_{X X} Z_{Y Y}-Z_{X Y}^{2}=0
$$

The exceptional integral manifold $N$ in these coordinates is not a solution of the equation in the usual sense; it consists of all planes passing through the point $(0,0,0) \in \mathbb{R}^{3}$.

The good news, however, is that up to local contact equivalence this is the only counterexample. We will demonstrate this in two steps. Note that this example has the property that $\mathcal{J}$ defines an integrable distribution. First we will show that any counterexample for which $\mathcal{J}$ is integrable is locally contact equivalent to the example above. Next we will show that Assertion 2 holds if $\mathcal{J}$ is not integrable. 
Proposition 3. Suppose that $\mathcal{J}$ is integrable, and let $F$ be an intermediate integral for $\mathcal{I}$ such that $d F$ never vanishes on $M$. If $N^{2}$ is an integral manifold of $\mathcal{K}$ but not of $\mathcal{I}$, then there exist local coordinates $x, y, z, p, q$ such that $\mathcal{I}$ is generated by the forms

$$
\begin{gathered}
\theta=d z-p d x-q d y, \\
d \theta=-d p \wedge d x-d q \wedge d y, \\
\Omega=d x \wedge d y,
\end{gathered}
$$

$F=z$, and $N$ is defined by the equations $z=p=q=0$.

Proof. Since $\mathcal{J}$ is integrable, the Frobenius theorem implies that locally there exist independent functions $X, Y, Z$ on $M$ such that

$$
\mathcal{J}=\{d X, d Y, d Z\}
$$

Moreover, the Pfaff theorem implies that these functions can be chosen so that up to a nonzero multiple,

$$
\theta=d Z-P d X-Q d Y
$$

for some functions $P, Q$ on $M$. Since $\theta \wedge(d \theta)^{2} \neq 0$, the functions $X, Y, Z, P, Q$ are independent and so form a local coordinate system on $M$. (See [1] for details.) Since $\Omega=\omega^{1} \wedge \omega^{2}$ with $\omega^{1}, \omega^{2} \in \mathcal{J}$, we must have $\Omega \equiv \lambda d X \wedge d Y \bmod \theta$ for some nonvanishing $\lambda$.

Now any function $F(X, Y, Z)$ is an intermediate integral for $\mathcal{I}$; conversely, every intermediate integral must have this form. For any such function, we can write

$$
d F=F_{Z} \theta+\left(F_{X}+P F_{Z}\right) d X+\left(F_{Y}+Q F_{Z}\right) d Y .
$$

If $N^{2}$ is an integral manifold of $\mathcal{K}$ but not of $\mathcal{I}$, then $N$ must be defined by the equations

$$
F=F_{X}+P F_{Z}=F_{Y}+Q F_{Z}=0 .
$$

Since $d F$ never vanishes, it must also be true that $F_{Z} \neq 0$ on $N$. In a neighborhood of $N$, define new coordinates $(x, y, z, p, q)$ by

$$
\begin{gathered}
x=X, \\
y=Y, \\
z=F(X, Y, Z), \\
p=F_{X}+P F_{Z}, \\
q=F_{Y}+Q F_{Z} .
\end{gathered}
$$

We have

$$
d z-p d x-q d y=F_{Z} \theta,
$$

so this change of variables is a contact transformation. Furthermore, $F=z$ and $N$ is defined by the equations $z=p=q=0$.

Theorem 4. Suppose that the system $\mathcal{J}$ is not integrable and that either

1. $d \theta \equiv 0 \bmod \mathcal{J}$, or

2. $\theta \wedge d \theta \wedge \Omega$ never vanishes.

Let $F$ be an intermediate integral for $\mathcal{I}$ such that $d F$ never vanishes on $M$. If $i: N^{2} \hookrightarrow M$ is any integral manifold of $\mathcal{K}$, then $N$ is an integral manifold of $\mathcal{I}$. 
The assumptions on $d \theta$ are essentially constant type assumptions; the first assumption holds for parabolic equations, and the second holds for hyperbolic or elliptic equations.

Proof. Choose 1-forms $\omega^{3}, \omega^{4}$ on $M$ so that $\left\{\theta, \omega^{1}, \omega^{2}, \omega^{3}, \omega^{4}\right\}$ is a basis for the cotangent space at each point of $M$. Since $F$ is an intermediate integral for $\mathcal{I}$, $d F$ must lie in the last derived system of $\mathcal{J}$. (See [1] for a discussion of derived systems.)

First suppose that assumption 1 holds. Then

$$
\left.\begin{array}{l}
d \theta \equiv 0 \\
d \omega^{1} \equiv r_{1} \omega^{3} \wedge \omega^{4} \\
d \omega^{2} \equiv r_{2} \omega^{3} \wedge \omega^{4}
\end{array}\right\} \quad \bmod \mathcal{J}
$$

where by the nonintegrability hypothesis $r_{1}, r_{2}$ never vanish simultaneously. By choosing a different basis for $\left\{\omega^{1}, \omega^{2}\right\}$ if necessary, we can assume that $r_{1} \equiv 0, r_{2} \neq$ 0 , so that

$$
\mathcal{J}^{(1)}=\left\{\theta, \omega^{1}\right\} .
$$

Now since $\theta$ is a contact form, $d \theta$ has no linear divisors modulo $\theta$. Therefore the expression $d \theta \bmod \mathcal{J}^{(1)}$ cannot vanish at any point of $M$. So if $\mathcal{J}^{(2)}=\left(\mathcal{J}^{(1)}\right)^{(1)}$ is nonzero, then it has dimension 1 and can be expressed in the form

$$
\mathcal{J}^{(2)}=\left\{\omega^{1}-s \theta\right\}
$$

for some function $s$. Therefore, any intermediate integral $F$ satisfies

$$
d F=a\left(\omega^{1}-s \theta\right)
$$

with $a \neq 0$, and the argument of [4] is valid.

Next suppose that assumption 2 holds. Then

$$
\left.\begin{array}{l}
d \theta \equiv r_{0} \omega^{3} \wedge \omega^{4} \\
d \omega^{1} \equiv r_{1} \omega^{3} \wedge \omega^{4} \\
d \omega^{2} \equiv r_{2} \omega^{3} \wedge \omega^{4}
\end{array}\right\} \quad \bmod \mathcal{J}
$$

where $r_{0}$ never vanishes. So $\mathcal{J}^{(1)}$ is 2-dimensional and can be expressed in the form

$$
\mathcal{J}^{(1)}=\left\{\omega^{1}-s_{1} \theta, \omega^{2}-s_{2} \theta\right\}
$$

for some functions $s_{1}, s_{2}$. Therefore, any intermediate integral $F$ satisfies

$$
d F=a\left(\omega^{1}-s_{1} \theta\right)+b\left(\omega^{2}-s_{2} \theta\right)
$$

where $a, b$ never vanish simultaneously, and the argument of [4] is valid.

\section{REFERENCES}

[1] R. Bryant, S. Chern, R. Gardner, H. Goldschmidt, and P. Griffiths, Exterior Differential Systems, Math. Sci. Res. Inst. Publ. 18, Springer-Verlag, New York, 1991. MR 92h:58007

[2] R. Bryant and P. Griffiths, Characteristic cohomology of differential systems II: Conservation laws for a class of parabolic equations, Duke Math J. 78 (1995) 531-676. MR 96d:58158

[3] E. Goursat, Leçons sur i'intégration des équations aux dérivées partielles du second ordre, vol. I, Gauthier-Villars, Paris, 1890.

[4] R. Hermann, Geometry, Physics, and Systems, Marcel Dekker, Inc., New York, 1973. MR 58:13104

Department of Mathematics, University of Colorado, Boulder, Colorado 80309

E-mail address: Jeanne.Clelland@Colorado.edu 